\title{
Examining the Relation between Heart Rate Variability, Flow, and Shooting in Wheelchair Athletes
}

\author{
Youngsook Kim ${ }^{1}$, Seunghyun Hwang ${ }^{2}$, Sanghoon Park ${ }^{3}$, Seongkwan Cho ${ }^{4}$, Eonho Kim ${ }^{5}$ \\ ${ }^{1}$ Department of Sport Science, Korea Institute of Sport Science, Seoul; ${ }^{2}$ Department of Leisure Sport, Kyungpook National University, Daegu; ${ }^{3}$ Department of Physical \\ Education, Yonsei University, Seoul, Korea; ${ }^{4}$ College of Nursing and Health Sciences, Texas A\&M International University, Laredo, TX, USA; ${ }^{5}$ Department of Physical \\ Education, Dongguk University, Seoul, Korea
}

PURPOSE: Heart rate variability leads affective changes, which are associated with athletic performance. This study aimed to examine the relationship between heart rate variability, athletic flow, and shooting performance in wheelchair shooting athletes.

METHODS: Eight male and female wheelchair shooting athletes were participated in the study. Heart rate variability was measured at rest and during shooting practice and competitions. Flow experience and shooting score were collected only during practice and actual shooting. SPSSWindows 25.0 was used to perform the following analysis. A repeated one-way ANOVA was applied to analyze the differences in heart rate variability at rest, during practice and actual shooting. In order to see the linear and curvilinear relationship among the variables, a hierarchical regression analysis was conducted.

RESULTS: The level of average heart rate and the ratio of Low Frequency (LF) to High Frequency (HF) of heart rate variability were significantly at different situations. At the resting period average heart rate was the highest while LF/HF was the lowest, which indicated that LF was less activated than $\mathrm{HF}$ at rest as compared to actual shooting. A hierarchical regression analysis showed that flow was a significant linear predictor for shooting performance.

CONCLUSIONS: Heart rate variability is changing depending on the circumstance that athletes faced to, which suggests that the changes may be associated with psychological states and performance.

Key words: Heart rate variability, Flow, Autonomic nerve system, Cardiac activity, Psychophysiology

\section{INTRODUCTION}

Among many psychophysiological changes of the human body, analysis of heart rate variability (HRV) has gained attention with regard to its relation with athletic performance [1]. HRV reflects the ability of the heart to respond and change in reaction to unexpected stimuli and environmental changes, and it evaluates the autonomic nervous system responsible for the regulation of cardiac activity [2]. As certain frequencies of heart rate (HR) are reportedly associated with and influence both the sympathetic and parasympathetic branches of the autonomic nervous system, HR is used to evaluate the activation of the systems [3].

$\mathrm{HRV}$ is measured in various parameters. In particular, HRV is ana- lyzed in the time domain, which reflects the RR interval, and in the frequency domain, which indicates the activity of the autonomic nervous system. For instance, the standard deviation of RR interval (SDNN) and the root mean square of successive differences (RMSSD), or the square root of the average of the squares of successive differences between adjacent NNs, are typical time domain parameters. For the frequency domain, spectrum analysis is used to analyze high frequency (HF, 0.15-0.4 $\mathrm{Hz}$ ), low frequency (LF, 0.04-0.15 Hz), and very low frequency (VLF, $0.003-0.04 \mathrm{~Hz}$ ) bands as well as the ratio between LF and HF (LF/HF). An individual's HRV is analyzed through such measurements to evaluate their psychophysiological changes. The time domain method involves the mathematical calculation of changes in RR intervals, or the

Corresponding author: Eonho Kim Tel +82-2-970-9566 Fax +82-2-970-9502 E-mail eonkimवsports.re.kr

Received 3 May 2019 Revised 20 May 2019 Accepted 21 May 2019

() This is an Open Access article distributed under the terms of the Creative Commons Attribution Non-Commercial License (http://creativecommons.org/licenses/by-nc/4.0/) which permits unrestricted non-commercial use, distribution, and reproduction in any medium, provided the original work is properly cited. 
intervals between heart beats. In particular, SDNN indicates the overall activity of the autonomic nervous system, with higher SDNN indicating higher resilience against stress and healthier status. RMSSD points to the activity of the parasympathetic nervous system; higher RMSSD indicates better cardiac stability [3]. In frequency domain methods employing spectrum analysis of each frequency band of cardiac activity, VLF and LF manifest the sympathetic and parasympathetic nervous systems, but mostly the activity of the sympathetic nervous system. Increases in LF demonstrate decreases in HRV and increases in anxiety and stress $[1,3]$. HF is regulated through the activity of the parasympathetic nervous system, and its increase reflects increases in HRV. In contrast, decreases in HF are associated with emotions related to stress, including anxiety and worry. LF/HF shows the overall balance of the autonomic nervous system. A high LF/HF signals that the sympathetic nervous system is relatively more active or that the parasympathetic nervous system is suppressed. When the sympathetic nervous system is relatively more activated, as in situations involving high arousal from anxiety or tension, the LF/HF ratio increases $[1,3]$. When the parasympathetic nervous system is relatively more activated, as in situations involving low arousal from helplessness or depression, the LF/HF ratio decreases $[1,3]$.

The autonomic nervous system is activated to respond to any changes in the environment and tasks, such as competition; its activation causes psychological and physical tension, as well as the resulting increase in HR. Such psychological arousal is associated with decreased parasympathetic activity and increased sympathetic activity, and this interaction causes changes in cardiac activity as well as emotional changes [4,5]. A high HRV indicates the ability of the autonomic nervous system to respond actively to stressful situations, and psychological relaxation reportedly increases when HRV is high [6,7]. Ortega \& Wang [8] reported that HRV can be an objective tool to quantify the subjective psychological states experienced by athletes participating in competitions as it is an indicator of emotional responses in sports science. They measured HRV, self-efficacy, mental skills, and shooting scores in 61 shooting athletes and tested the relation between these variables. Their analysis was conducted for three groups: beginner, intermediate, and advanced shooting athletes. HRV, self-efficacy, mental skills, and predicted scores were documented while the participants were at rest prior to the start of preliminary rounds. HRV had a significant correlation with self-efficacy and also significantly predicted shooting scores. In a regression model predicting shooting scores, HRV was the only significant variable. SDNN of advanced shooting athletes was higher than that of the other two groups, and advanced shooting athletes also had significantly lower HR. Moreover, advanced shooting athletes were reported to have used more selftalk and relaxation. As suggested by Ortega \& Wang [8], HRV would serve as a snapshot of the pre-competition psychological states of athletes. The present study aimed to observe changes in HRV before and during competition along with flow experience.

Flow, which was first proposed by Csikszentmihalyi [9], refers to a psychological state where an individual deeply focuses on a behavior and forgets their senses of time, space, and self. Flow is studied as a predictor of successful athletic performance [10]. As for sport performance, it is defined as the optimal moment at which an athlete's mind and body unite during practice or competition $[11,12]$. Research on flow has begun to connect physiological responses and subjective flow experience. The studies exploring the relationship between flow and physiological responses, such as sympathetic and parasympathetic activities, and stress responses during motor and cognitive tasks $[13,17]$. For example, Peifer and colleagues [17] measured HRV, flow, salivary cortisol, and stress from twenty-two college students while they were performing a computer simulation game for 60 minutes. They found a positive linear relationship between HF-HRV (parasympathetic nervous system) and flow experience, an inverted-U relationship of LF-HRV (sympathetic nervous system) and cortisol with flow experience. The activation of the sympathetic nervous system and cortisol secretion initially increase as flow experience increases, but when they are excessive, flow experience decreases. The autonomic nervous system dynamically changes as to responds to environmental demands, such as training of new skills and competition.

Taken together, athletes experience changes in HRV, which may lead a change in psychological state. Compare to other sports, shooting does not require a large amount of physical load; however, shooting athletes experience a large amount of psychological and physiological changes when they are in a competitive circumstance. Therefore, it is important to see the differences of HRV and flow experience in different conditions, and the relation to shooting performance. This study aimed to measure HRV and flow experience in shooting and to test the relationships with wheelchair shooting athletes. In particular, the study tested the differences in HRV at rest and during actual shooting, and examined the linear or non-linear (curvilinear) relations between HRV during actual shooting, perceived flow, and shooting score. The hypotheses were set as followings: 1) HRV (LF, HF, LF/HF, SDNN, RMSSD) would be different due to the conditions. 2) HRV and flow could be a linear or curvilinear predictor of shooting scores. 3) HRV would be related to flow. 


\section{METHODS}

\section{Participants}

Ten wheelchair shooting elite athletes participated in the study, and all they had experience playing in a National Team. Values obtained from HR monitor are sometimes abnormal because HRV measures are sensitive in responding to environmental demands. The criteria by Nunan er al. [18] was used to validate the obtained data. Abnormal data were identified and discarded. Only data from eight athletes' data was used for analysis. Five were male and three were female athletes aging from 31 to 56 years. Their average career as a shooting athlete was thirteen years with 7.02 of standard deviation.

\section{Procedures}

After obtaining approval from the Institutional Review Board, we contacted a team with interest in participation and recruited athletes who wished the participation. Precautions were explained to the athletes, and their consent was subsequently obtained. The athletes were first asked to rest and to wear a Polar HR belt (Polar-R5800) on their chest. They gave verbal confirmations that they experienced no discomfort, and connectivity with a Polar watch enabling real-time monitoring was confirmed.

After all of the instruments were ready, the participants' HR and HRV were measured via the Polar HR belt at rest for five minutes followed by the practice shooting for 15 minutes to calibrate shooting. Then, the athletes should make 60 shots in 1 hour and 15 minutes. Since ten shots comprised one series, HRV and flow experience were measured separately for the six series. A research assistant behind the participants manipulated the Polar watch to measure HRV and separate each series during the shooting, and verbally asked their flow experience after each series while being careful not to interfere with their performance.

\section{Instruments \\ 1) HRV}

To measure the participants' HR and HRV, Polar-R5800 was used, which non-invasively collects HR information through EKG sensors at a sampling rate of 1,000 Hz. The R-R interval data measured through Polar-R5800 was transferred to Polar ProTrainer 5, a computer program developed by Polar, to save data for HR and HRV. HR and HRV data collected through Polar are reliable and valid and are being used in many studies [19]. HRV was analyzed in the time and frequency domains. In the time domain, RMSSD, SDNN and in the frequency domain, LF, HF, and LF/HF. LF were used for HRV parameters.

\section{2) Flow}

To measure the athletes' perceived flow experience, the Flow Short Scale developed by Engeser \& Rheinberg [20] was revised to make it more applicable for shooting athletes and it consisted of the following four questions: 1) I experienced flow in this series between aiming and firing. 2) All of my movements during the series were natural. 3) I was able to focus well during this series. 4) I felt that I was able to control all of my movements during this series. An 11-point Likert scale (0: Completely disagree to 5: Neutral to 10: Completely agree) was used. Table 1 describes the mean and internal consistency of the questionnaire. The mean scores of the questions ranged between 6.40 and 7.15, and the internal consistency represented by Cronbach's a was .858, which is similar to the original scale's internal consistency (.87).

Table 2. Shooting performance scores

\begin{tabular}{lcccc}
\hline Series & Mean & $\begin{array}{c}\text { Standard } \\
\text { deviation }\end{array}$ & Skewness & Kurtosis \\
\hline Practice shooting & 9.68 & 0.18 & 0.488 & 0.421 \\
Series 1 & 9.65 & 0.23 & 0.090 & -1.613 \\
Series 2 & 9.63 & 0.25 & -0.304 & 0.146 \\
Series 3 & 9.60 & 0.21 & -0.935 & 0.350 \\
Series 4 & 9.45 & 0.28 & -0.587 & -1.106 \\
Series 5 & 9.35 & 0.14 & 0.404 & -0.229 \\
Series 6 & 9.35 & 0.26 & 0.611 & -0.021 \\
Overall mean & 9.53 & 0.25 & -0.137 & -1.241 \\
\hline
\end{tabular}

Table 1. Descriptive statistics of the measure of flow experience

\begin{tabular}{|c|c|c|c|c|c|}
\hline Question & Mean & $\begin{array}{l}\text { Standard } \\
\text { Deviation }\end{array}$ & Skewness & Kurtosis & $\begin{array}{c}\text { Internal } \\
\text { Consistency }\end{array}$ \\
\hline 1. I experienced flow in this series between aiming and firing. & 7.06 & 1.41 & -0.211 & 0.378 & \multirow{5}{*}{ Cronbach's $a=.858$} \\
\hline 2. All of my movements during the series were natural. & 7.15 & 1.50 & -0.180 & -0.399 & \\
\hline 3. I was able to focus well during this series. & 6.70 & 1.56 & 0.441 & -0.622 & \\
\hline 4. I felt that I was able to control all of my movements during this series. & 6.40 & 1.71 & 0.412 & -0.117 & \\
\hline Overall mean & 6.83 & 1.29 & 0.206 & -0.063 & \\
\hline
\end{tabular}




\section{3) Shooting Performance}

Electronic shooting target was used to confirm the score and the number of firings in real time. The maximum score was 10 , but there were additional decimal scores (e.g., 10.9). The score for each shot was recorded electronically, and the mean score of 10 shots from each series was used (Table 2).

\section{Statistical Analysis}

The data were collected on Excel where abnormal data was deleted by Nunan, Sandercock, and Brodie' normal range of HRV [18]. An excessive high or low HRV based on the Nunan's criteria was not included for the analysis. Subsequently, SPSS Windows 25.0 was used to calculate descriptive statistics. The following statistical analyses were conducted for each research task. A repeated measures ANOVA was used to analyze the differences in HRV at rest, during practice and actual shooting. The overall mean value of the six series was used to assess HRV during the actual shootin. Tukey's post-hoc analysis was conducted when a significant difference was found. In order to see the linear and (or) non-linear relationship between variables during actual shooting, a hierarchical regression analysis was tested, using 48 cases (six series times eight participants), through the goodness of fit of block 1: $y=\beta_{0}+\beta_{1} x_{1}+\beta_{2} x^{2}{ }_{1}+e$ and block 2: $y=\beta_{0}+\beta_{1} x_{1}+\beta_{2} x^{2}+e$ as suggested by Abraham \& Ledolter [21]. All statistical significance was set at 0.05 .

\section{RESULTS}

\section{Difference in HRV during rest, practice shooting, and} actual shotting

The descriptive statistics of HRV are presented in Table 3. Table 4 shows the statistical differences in HRV during rest, practice and actual shooting. The repeated measures ANOVA of the average HR was statistically signifi- cant, $F(2,7)=3.925$, which effect size $\left(\right.$ partial $\left.\eta^{2}\right)$ was .38 . Post-hoc analyses revealed that the average $\mathrm{HR}$ at rest was significantly larger than that during practice shooting ( $p=.04$, CI 95\%: 1.87-58.12). Another significant difference was found in LF/HF, F $(2,7)=6.200$, which effect size $\left(\right.$ partial $\left.\eta^{2}\right)$ was .47. Post-hoc analyses revealed that LF/HF at rest was significantly less than that during practice shooting ( $p=.03$, CI 95\%: -349.84 -22.80) and that during actual shooting ( $p=.02$, CI 95\%: -420.58 -46.43).

\section{Relationship among shooting score, HRV, and flow Figures, Tables, and Schemes}

A hierarchical regression analysis was used to test linear and curvilin-

Table 3. Descriptive statistics of HRV during rest, practice shooting, and actual shooting

\begin{tabular}{llrr}
\hline HRV & Condition & Mean & $\begin{array}{r}\text { Standard } \\
\text { Deviation }\end{array}$ \\
\hline Average HR (ms) & Rest & 747.13 & 1.25 \\
& Practice & 717.13 & 33.69 \\
& Shooting & 709.33 & 51.31 \\
RMSSD (ms) & Rest & 26.44 & 3.32 \\
& Practice & 24.81 & 4.77 \\
& Shooting & 24.90 & 5.27 \\
SDNN (ms) & Rest & 49.74 & 1.24 \\
& Practice & 55.60 & 13.21 \\
VLF $\left(\mathrm{ms}^{2}\right)$ & Shooting & 49.45 & 8.84 \\
& Rest & $1,820.29$ & 210.88 \\
LF $\left(\mathrm{ms}^{2}\right)$ & Practice & $3,489.24$ & $1,892.30$ \\
& Shooting & $2,831.24$ & $1,092.29$ \\
& Rest & 734.07 & 129.53 \\
HF $\left(\mathrm{ms}^{2}\right)$ & Practice & $1,017.10$ & 289.20 \\
& Shooting & $1,048.72$ & 436.67 \\
& Rest & 264.85 & 8.30 \\
LF/HF & Practice & 252.65 & 91.88 \\
& Shooting & 252.69 & 94.41 \\
& Rest & 278.69 & 55.54 \\
& Practice & 465.01 & 205.78 \\
& Shooting & 512.19 & 257.37 \\
\hline & & & \\
& & & \\
& & &
\end{tabular}

Table 4. Results of a repeated measures ANOVA

\begin{tabular}{|c|c|c|c|c|c|c|}
\hline & Sum of Squares & Degree of Freedom & Mean of Squares & $F$ & $p$ & Post-hoc Analysis \\
\hline Average HR (ms) & $6,370.454$ & 2 & $3,185.227$ & $3.925^{*}$ & .044 & $1>2$ \\
\hline RMSSD (ms) & 13.382 & 2 & 6.691 & 0.506 & .613 & \\
\hline SDNN (ms) & 192.588 & 2 & 96.294 & 1.397 & .280 & \\
\hline $\operatorname{VLF}\left(\mathrm{ms}^{2}\right)$ & $11,307,696$ & 2 & $5,653,848$ & 3.131 & .075 & \\
\hline $\mathrm{LF}\left(\mathrm{ms}^{2}\right)$ & $480,292.03$ & 2 & 240,146 & 2.985 & .083 & \\
\hline $\mathrm{HF}\left(\mathrm{ms}^{2}\right)$ & 791.99 & 2 & 395.995 & 0.105 & .901 & \\
\hline $\mathrm{LF} / \mathrm{HF}$ & $243,915.32$ & 2 & $121,957.7$ & $6.2^{*}$ & .012 & $1<2=3$ \\
\hline
\end{tabular}

Post-hoc analysis: 1 = at rest, 2 = during practice shooting, $3=$ during actual shooting. ${ }^{*} p<.05,{ }^{* *} p<.01$. 
ear relations. In block $1\left(y=\beta_{0}+\beta_{1} x_{1}+e\right)$, only the raw scores of the predictor variable were entered. In block $2\left(y=\beta_{0}+\beta_{1} x_{1}+\beta_{2} x_{1}{ }_{1}+e\right)$, squares of the predictor variable were additionally entered to test for goodness of fit of the model as well as the statistical significance of each coefficient $\left(\beta_{1}\right.$ and $\left.\beta_{2}\right)$.

To see the predictability of HRV for shooting performance, HRV was set as the predictor variable $(x)$, and shooting score was used as the outcome variable $(y)$. The linear and curvilinear relations between HRV and shooting score were both non-significant (Table 5). And the relations between HRV and flow were not found (Table 6). Those results indicated that changes in HRV during shooting were not associated with changes in shooting performance and flow experience.

However, a significant relationship was found in flow and shooting performance. Although both the linear and curvilinear models were sig- nificant, the change in $\mathrm{R}^{2}$ from model 1 to 2 was very small (Table 6). Thus, flow linearly predicted shooting performance, and the regression coefficient was .57 .

\section{DISCUSSION}

This study explored the HRV, flow experience, and shooting performance before and during shooting of wheelchair shooting athletes. Differences in HRV in different settings were tested at rest, during practice and actual shooting. Hierarchical regression analyses were conducted to analyze linear and non-linear (curvilinear) relationships.

Regarding HRV under different conditions, the average HR was higher at rest as compared to the average HR during practice and actual

Table 5. Linear and curvilinear predictability of HRV and flow for shooting performance

\begin{tabular}{|c|c|c|c|c|c|c|c|}
\hline Predictor & Model & $\beta_{1}$ & $\beta_{2}$ & $R^{2}$ & $F$ & $d f 1$ & $d f 2$ \\
\hline \multirow[t]{2}{*}{ Average HR (ms) } & Model 1 (Linear) & 0.14 & - & .018 & 1.00 & 1 & 46 \\
\hline & Model 2 (Curvilinear) & -1.15 & 1.28 & .022 & 0.58 & 1 & 45 \\
\hline \multirow[t]{2}{*}{ RMSSD (ms) } & Model 1 (Linear) & 0.16 & - & .026 & 1.42 & 1 & 46 \\
\hline & Model 2 (Curvilinear) & -0.61 & 0.77 & .034 & 0.93 & 1 & 45 \\
\hline \multirow[t]{2}{*}{ SDNN (ms) } & Model 1 (Linear) & -0.04 & - & .001 & 0.07 & 1 & 46 \\
\hline & Model 2 (Curvilinear) & -1.55 & 1.52 & .035 & 0.97 & 1 & 45 \\
\hline \multirow[t]{2}{*}{$\operatorname{VLF}\left(m s^{2}\right)$} & Model 1 (Linear) & -0.23 & - & .052 & 2.97 & 1 & 46 \\
\hline & Model 2 (Curvilinear) & 0.27 & -0.52 & .071 & 2.03 & 1 & 45 \\
\hline \multirow[t]{2}{*}{$\operatorname{LF}\left(m s^{2}\right)$} & Model 1 (Linear) & -0.08 & - & .006 & 0.3 & 1 & 46 \\
\hline & Model 2 (Curvilinear) & -0.04 & -0.04 & .006 & 0.15 & 1 & 45 \\
\hline \multirow[t]{2}{*}{$\mathrm{HF}\left(\mathrm{ms}^{2}\right)$} & Model 1 (Linear) & -0.16 & - & .026 & 1.46 & 1 & 46 \\
\hline & Model 2 (Curvilinear) & 0.33 & -0.51 & .042 & 1.15 & 1 & 45 \\
\hline \multirow[t]{2}{*}{$\mathrm{LF} / \mathrm{HF}$} & Model 1 (Linear) & 0.15 & - & .024 & 1.31 & 1 & 46 \\
\hline & Model 2 (Curvilinear) & -0.36 & 0.53 & .042 & 1.17 & 1 & 45 \\
\hline \multirow[t]{2}{*}{ Flow } & Model 1 (Linear) & $.57^{* * *}$ & - & .327 & $22.39^{* *}$ & 1 & 46 \\
\hline & Model 2 (Curvilinear) & -.39 & .97 & .342 & $11.69^{* *}$ & 1 & 45 \\
\hline
\end{tabular}

${ }^{*} p<.05,{ }^{* *} p<.01$.

Table 6. Linear and curvilinear predictability of HRV for flow

\begin{tabular}{|c|c|c|c|c|c|c|c|}
\hline Predictor & Model & $\beta_{1}$ & $\beta_{2}$ & $R^{2}$ & $F$ & $d f 1$ & $d f_{2}$ \\
\hline \multirow[t]{2}{*}{ Average HR (ms) } & Model 1 (Linear) & 0.12 & - & .015 & 0.70 & 1 & 46 \\
\hline & Model 2 (Curvilinear) & -1.55 & 1.68 & .020 & 0.47 & 1 & 45 \\
\hline \multirow[t]{2}{*}{ RMSSD (ms) } & Model 1 (Linear) & 0.07 & - & .005 & 0.25 & 1 & 46 \\
\hline & Model 2 (Curvilinear) & -0.51 & 0.58 & .010 & 0.22 & 1 & 45 \\
\hline \multirow[t]{2}{*}{ SDNN (ms) } & Model 1 (Linear) & -0.1 & - & .010 & 0.48 & 1 & 46 \\
\hline & Model 2 (Curvilinear) & -0.06 & -0.04 & .010 & 0.24 & 1 & 45 \\
\hline \multirow[t]{2}{*}{$\operatorname{VLF}\left(m s^{2}\right)$} & Model 1 (Linear) & -0.21 & - & .045 & 2.18 & 1 & 46 \\
\hline & Model 2 (Curvilinear) & 0.52 & -0.76 & .085 & 2.09 & 1 & 45 \\
\hline \multirow[t]{2}{*}{$\operatorname{LF}\left(m s^{2}\right)$} & Model 1 (Linear) & -0.04 & - & .001 & 0.06 & 1 & 46 \\
\hline & Model 2 (Curvilinear) & -1.03 & 1.02 & .041 & 0.97 & 1 & 45 \\
\hline \multirow[t]{2}{*}{$\mathrm{HF}\left(\mathrm{ms}^{2}\right)$} & Model 1 (Linear) & -0.14 & - & .019 & 0.89 & 1 & 46 \\
\hline & Model 2 (Curvilinear) & 0.13 & -0.28 & .023 & 0.53 & 1 & 45 \\
\hline \multirow[t]{2}{*}{$\mathrm{LF} / \mathrm{HF}$} & Model 1 (Linear) & 0.11 & - & .011 & 0.52 & 1 & 46 \\
\hline & Model 2 (Curvilinear) & -0.69 & 0.82 & .053 & 1.27 & 1 & 45 \\
\hline
\end{tabular}


shooting. Although the average HR increases under physical or psychological workload in general [22], the observed difference in the average HR of shooting athletes is interesting. Shooting involves much higher mental stress. Moreover, since very small physical movements may hinder their performance, athletes try to control physiological responses, such as breathing and heart beating. The average HR at rest was higher than actual shooting as shown in our data. More interestingly, changes in HRV (LF/HF) were found across three different conditions in this study. The LF/HF during shooting was statistically higher than at rest, which indicates that the sympathetic nervous system is relatively more active or that the parasympathetic nervous system is suppressed. When the sympathetic nervous system is relatively more activated, the LF/HF ratio increases, which is lined to higher anxiety and psychological tension [3]. This result may suggest that para- and sympathetic nervous system represented by HF and LF in HRV deliver a more meaning source than the time interval of heart beating (i.e., HR) to consider mental arousal when assuming the actual shooting generate more stress in our body. Similar results about the relationship between LF/HF and mental stress were found in other studies. Morales et al. [23] reported higher LF, LF/HF, cognitive anxiety, and physical anxiety of Judo athletes during official competitions than during unofficial competitions. Blásquez et al. [24] also reported that LF/HF increases in swimmers during competition compared with during practice. Similarly, decrease in HF among volleyball athletes was observed when a competition was approaching [25]. A competition involves mental workload and stress. Such increased stress involves a transition from sympathetic nervous system activities to vagus nerve activities in the parasympathetic nervous system, which leads to decreased HF and increased LF/HF [26].

The predictability of HRV has been reported for motor performance [27-29]. The present work also explored how changes in HRV are related to flow experience and shooting performance. Only flow was a significant predictor for shooting performance. HRV did not show any relationship with shooting performance and flow, which is unexpected. Ortega \& Wang [8] found that SDNN among HRV parameters, self-efficacy, and mental skills positively predicted shooting performance. Moreover et al. [28] reported that the parasympathetic nervous system, and mental coping capabilities positively predicted dart throwing performance. As such, attention to the relationship between HRV and athletic performance is growing in recent years.

An important point of this study is to test a non-linear relation (i.e., inverted $\mathrm{U}$ relation) between HRV, flow, and shooting performance as suggested by Abraham \& Ledolter [21]. Unfortunately, none of non-linear relationship was found in this study. However, in a computer-based cognitive task, LF had an Inverted-U relation with flow, whereas HF has a positive linear relation with flow [17]. Manzano et al. [15] reported that increases in flow were associated with increases in overall HRV and LF/ HF. Like this study, Peifer et al. [17] and Manzano et al. [15] also were unsuccessful to see the significant relationship between HRV and task performance, but they nonetheless suggested the possibility of research on activation of the sympathetic-parasympathetic nervous systems and their relations with mental flow.

A limitation of this study is on the number of samples. There may be a disadvantage in validating the statistical results from small sample size. Measuring heart rate could be another limitation. We set up the stable environment as much as possible; however measurement error might be involved in our data. The following study should consider those limitation to help validating research outcome. It is unfortunate not to find out any significant relationship between HRV, flow and performance. We only found out the significant change in HRV due to the conditions, and the linear prediction of flow to performance. This study could be limited in interpreting the result, however this study guides a new approach to deal with mental and physiological state and performance at the same time. This study has contributed to the growing body on HRV and elite athletic performance. In particular, individual zone of optimal functioning model [30] seeks to quantify the emotional status of an individual, and identify his or her optimal zone for peak performance. The frame of HRV study could be integrated in finding optimal zone of individual performance. The HRV parameter of an individual could be used to identify the zone for peak performance. In addition to the theoretical development, this study may contribute to developing a device measuring mental state by physiological parameters such as HRV in relation to human performance [31].

\section{CONFLICT OF INTEREST}

No potential conflict of interest relevant to this article was reported.

\section{REFERENCES}

1. Morgan SJ, Mora JAM. Effect of heart rate variability biofeedback on sport performance, a systematic review. Appl Psychophysiol Biofeedback. 2017;42(3):235-45. 
2. Acharya UR, Joseph KP, Kannathal N, Min LC, Suri JS. Advances in cardiac signal processing; Springer 2007. p. 121-165.

3. Shaffer F, Venne J. Heart rate variability anatomy and physiology. Biofeedback. 2013;41:13-25.

4. Aubert AE, Seps B, Beckers F. Heart rate variability in athletes. Sports Med. 2003;33(12):889-919.

5. Berntson GG, Cacioppo JT. Heart rate variability: Stress and psychiatric conditions. In Dynamic electrocardiography, Malik M, Camm JA. Futura 2004. p. 57-64.

6. Lin IM, Tai LY, Fan SY. Breathing at a rate of 5.5 breaths per minute with equal inhalation-to-exhalation ratio increases heart rate variability. Int J Psychophysiol. 2014;91(3):206-11.

7. Wheat AL, Larkin KT. Biofeedback of heart rate variability and related physiology: A critical review. Appl Psychophysiol Biofeedback. 2010; 35(3):229-42.

8. Ortega E, Wang, CJK. Pre-performance Physiological State: Heart Rate Variability as a Predictor of Shooting Performance. Appl Psychophysiol Biofeedback. 2018;43(1):75-85.

9. Csikszentmihalyi M. Flow and the psychology of discovery and invention. Harper Collins 1997.

10. Jackson SA, Kimiecik JC, Ford SK, Marsh HW. Psychological correlates of flow in sport. J Sport Exerc Psychol. 1998;20(4):358-78.

11. Nakamura J, Csikszentmihalyi M, Snyder CR, Lopez SJ. Handbook of Positive Psychology. Oxford University Press 2002.

12. Scanlan DJ, Hess WR, Partensky F, Newman J, Vaulot D. High degree of genetic variation in Prochlorococcus (Prochlorophyta) revealed by RFLP analysis. Eur J Phycol. 1996;31(1):1-9.

13. Keller J, Bless H, Blomann F, Kleinböhl D. Physiological aspects of flow experiences: Skills-demand-compatibility effects on heart rate variability and salivary cortisol. J Exp Soc Psychol. 2011;47(4):849-52.

14. Kivikangas JM. Psychophysiology of flow experience: An explorative study. Master's Thesis, Helsinki: University of Helsinki 2006.

15. Manzano OD, Theorell T, Harmat L, Ullen F. The psychophysiology of flow during piano playing. Emotion. 2010;10(3):301-11.

16. Nacke LE, Nacke A, Lindley CA. Brain training for silver gamers: effects of age and game form on effectiveness, efficiency, self-assessment, and game play experience. Cyberpsychol Behav. 2009;12(5):493-99.

17. Peifer C, Schulz A, Schächinger H, Baumann N, Antoni CH. The relation of flow-experience and physiological arousal under stress-can $\mathrm{u}$ shape it? J Exp Soc Psychol. 2014;53:62-9.
18. Nunan D, Sandercock GR, Brodie DA. A quantitative systematic review of normal values for short-term heart rate variability in healthy adults. Pacing Clin Electrophysiol. 2010;33(11):1407-17.

19. Goodie JL, Larkin KT, Schauss S. Validation of Polar heart rate monitor for assessing heart rate during physical and mental stress. J Psychophysiol. 2000;14(3):159-64.

20. Engeser S, Rheinberg F. Flow, performance and moderators of challenge-skill balance. Motiv Emot. 2008;32(3):158-72.

21. Abraham B, Ledolter J. Statistical methods of forecasting. John Wiley and Sons 1983.

22. Hammoud S, Karam R, Mourad R, Saad I, Kurdi M. Stress and heart rate variability during university final examination among Lebanese students. Behav Sci. 2018;9(1):3.

23. Morales J, Garcia V, García-Massó X, Salvá P, Escobar R. The use of heart rate variability in assessing precompetitive stress in high-standard judo athletes. Int J Sports Med. 2013;34(2):144-51.

24. Blásquez JCC, Font GR, Ortís LC. Heart-rate variability and precompetitive anxiety in swimmers. Psicothema. 2009;21(4):531-6.

25. D’Ascenz F, Alvino F, Natali BM, Cameli M, Palmitesta P, et al. Precompetitive assessment of heart rate variability in elite female athletes during play offs. Clin Physiol Funct Imaging. 2014;34(3):230-6.

26. Hjortskov N, Rissen D, Blangsted AK, Falletin D, Lundberg U, et al. The effect of mental stress on heart rate variability and blood pressure during computer work. Eur J Appl Physiol. 2004;92(1-2):84-89.

27. Morgan SJ, Mora JAM. Effect of heart rate variability biofeedback on sport performance, a systematic review. Appl Psychophysiol Biofeedback. 2017;42(3):235-45.

28. Mosley E, Laborde S, Kavanagh E. The contribution of coping related variables and cardiac vagal activity on the performance of a dart throwing task under pressure. Physiol Behav. 2017;179:116-25.

29. Neumann DL, Thomas PR. Cardiac and respiratory activity and golf putting performance under attentional focus instructions. Psychol Sport Exerc. 2011;12(4):451-9.

30. Hanin YL. Individual zones of optimal functioning (IZOF) model: emotion-performance relationships in sport. In Emotions in sport Champaign, Hanin YL. Human Kinetics 2000.

31. Zangróniz R, Martínez-Rodrigo A, López MT, Pastor JM, FernándezCaballero A. Estimation of mental distress from photoplethysmography. Appl Sci. 2018;8(1):69. 